\title{
Design \& Implementation of 3-Bit High Speed Flash ADC for Wireless LAN Applications
}

\author{
Mirza Nemath Ali Baig ${ }^{1}$, Rakesh Ranjan ${ }^{2}$ \\ Assistant Professor, ECE dept., CMR Engineering College, Hyderabad, India 1,2
}

\begin{abstract}
Analog-to-digital (ADC) converter has become a very important part of electronics in the current digital world as they have a wide variety of applications. Among all the ADC's available, the Flash ADC is one of the fastest ADC but a main drawback of this ADC is its power consumption. So, the main objective of this paper is to implement a high speed low power Flash ADC. A design with 3-bit resolution has been implemented using seven OTA based comparators with a reference voltage of $250 \mathrm{mV}$ and a high speed encoder have been implemented using four full adders upon which the integration of different block ADC has been designed. All the circuits are simulated using $180 \mathrm{~nm}$ technology in Cadence Virtuoso Design environment. The supply voltage is 1.8v.Analog output of each comparator depending upon the comparison between the input and the reference voltage is fed to the encoder and finally the compressed digital output is obtained.
\end{abstract}

Keywords: Flash ADC, Comparator, OTA, Thermometer to Binary encoder, CMOS 180 nm Technology.

\section{INTRODUCTION}

With the advancement of technology, digital signal processing has progressed dramatically in recent years. Signal processing in digital domain provides high level of accuracy, low power consumption and small silicon area besides providing flexibility in design and programmability. The design process is also quite faster and cost effective. Furthermore, their implementation makes them suitable for integration with complex digital signal processing blocks in a compatible low-cost technology, particularly CMOS. In the end, it leads us to design a very high speed as well as systems with small die area called System on a chip (SoC), with a smaller number of chips using increased integration level.

This has resulted in the requirement of smart converters between analog and digital signals to cope up with the evolution of technology. Recent in many wireless mobile applications demand very high speed data converters with wide bandwidth, higher signal to noise ratio and variable (adaptive) resolution with optimized power and cost effectiveness. So there is a need for upgrading the performance of data converters to meet the demands of emerging technologies. So it is a challenging issue in the mixed signal design to have high speed, variable resolution data converters with less space and low power consumption.

Digital signal processing has been proved to be a robust and cost effective way of signal processing. In many applications the input and output signals of the system are inherently analog, so a conversion between analog and digital is needed at the interfaces [4]. A/D converter implements the interface between the real word analog signal and the DSP function block [7]. The examples of applications of A/D converters are video-imaging systems, personal communication systems. Higher resolution and higher speed A/D converter is required for applications, such as wireless communication, image processing etc.

\section{RELATED WORK}

A survey on flash ADC based on certain parameters like reference voltage, supply voltage resolution, Signal to Noise ratio and power consumption has been carried out. Descriptions of some papers are described below.

- Dhrubajyoti\&Sagar introduced a Low Power type ADC (Analog-to-Digital Converter) where the conventional comparators have been replaced with the CMOS inverter based comparator designs. The reported structure of the ADC is designed using 180nm technology and it consumes $130.9 \mu \mathrm{Watt}$ of average power while operating with an input frequency of $30 \mathrm{MHz}$, and a supply voltage of 1.8 Volt. Moreover, with the aim of increasing the design efficiency, an optimized analog layout, which occupies an area of $0.068 \mathrm{~mm}^{2}$, has been presented.[6]

AditiKar and Alaktold about an area efficient low power high Speed 3-bit Flash Type ADC using bit referenced encoder is proposed in $180 \mathrm{~nm} \mathrm{CMOS}$ technology. The concept of Threshold Modified Comparator Circuit (TMCC) is also introduced as a modification of the conventional comparator. The proposed design of the ADC occupies an active area of $0.0036 \mathrm{~mm} 2$ and consumes $43.146 \mu \mathrm{W}$ of Average Power while operating with an input frequency (fin) of $10 \mathrm{MHz}$ and a supply voltage of 1.8 Volt.[3]

- $\quad$ Pradeep and Amit give the description of the the design and implementation of a Low Power 3-bit flash Analog to Digital converter (ADC). It includes 7 
comparators and one thermometer to binary encoder. It is A block diagram of a conventional flash ADC is shown in implemented in 0.18um CMOS Technology. The pre- Fig.1. It has $2^{\mathrm{N}}-1$ comparators corresponding to $2^{\mathrm{N}}-1$ simulation of ADC is done in T-Spice. The response time quantization steps [14]. The total $2^{\mathrm{N}}$ resistors generate all of the comparator equal to $6.82 \mathrm{~ns}$ and for Flash $\mathrm{ADC}$ as the reference voltages. The comparator outputs one if the $18.77 \mathrm{~ns}$.The Simulated result shows the power input voltage is larger than the related reference voltage, consumption in Flash ADC as is $36.273 \mathrm{mw}$.The chip area and vice versa.

is for Flash ADC is 1044um2.[16]

The prime focus of my work is to develop a high speed flash ADC used in applications of Wireless LAN systems. The main aim of this paper is to implement of a high speed ADC used for the application by Integrating resistor ladder, comparators and thermometer to binary code encoder, flash ADC is designed. All the parameters of ADCs are tested and tradeoff between speed and power is made. A detailed analysis of the implementation is done and compared the proposed ADC with other types of ADCs used for the specific application. The complete ADC is designed and implemented using CMOS $180 \mathrm{~nm}$ technology using CADENCE Virtuoso Design environment.

\section{III.FLASH ADC}

For high speed applications, flash ADC is often used. Flash ADC is very fast and used for low resolution application due to its parallel architecture. It is best and less complex up to 8 bits. But when the number of bits increased complexity increases since the number of comparator needed is large. The main significance of flash $\mathrm{ADC}$ is that they are used within pipeline and sigma delta converters.

Comparators are the key analog building block of any flash ADC and strongly influence performance. A high degree of comparator accuracy is essential for good ADC performance. However, integration of analog circuitry in low voltage scale VLSI technologies results in degraded precision due to large device mismatch and limited voltage swing [6]. Analog offset correction techniques are typically used, but these schemes are increasingly difficult to implement in modern CMOS processes. For this reason, the issue of comparator offset is becoming a bottleneck in the design of flash ADCs.

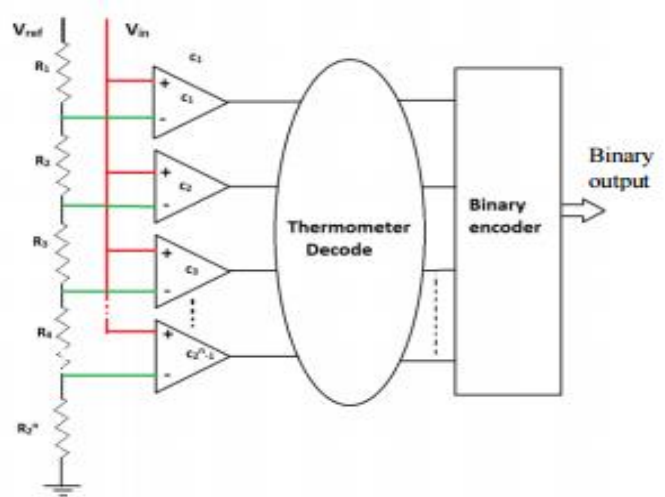

Fig.1Conventional flash ADC
The reference voltages are typically generated through the use of a resistor ladder. The thermometer decode block generates a " 1 of $n$ " code which is converted to binary.

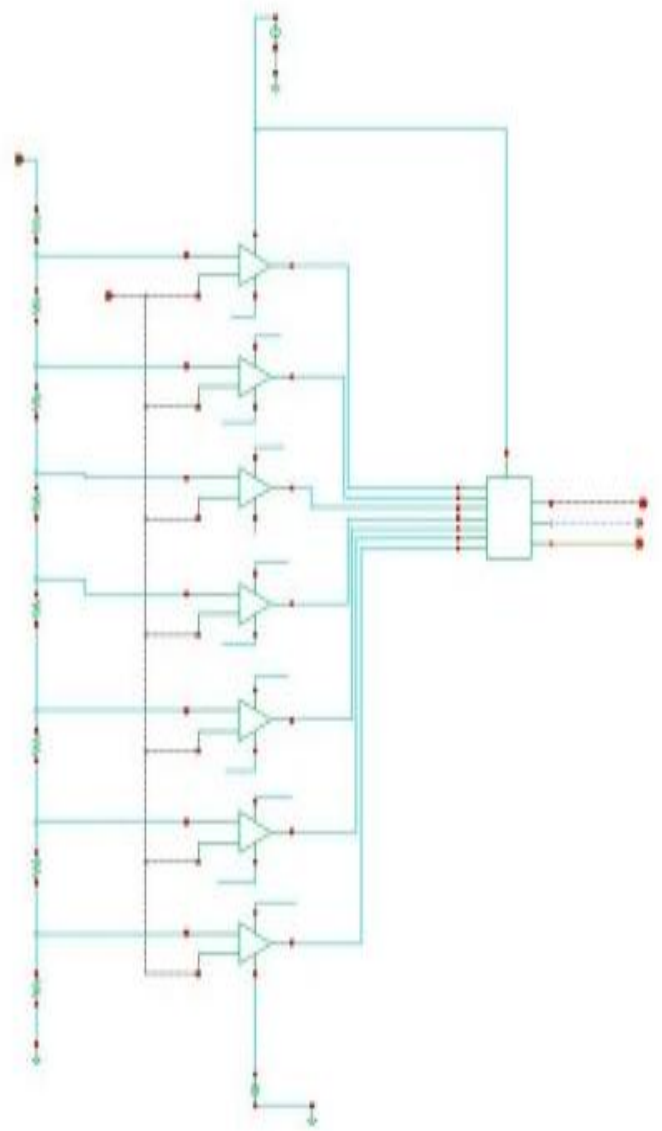

Fig. 2Schematic of Three bit Flash ADC Implementation

As shown in fig. 2 Schematic of Three bit Flash ADC Implementation consists of the three blocks (resistor ladder, 7 comparators and thermometer code to binary code converter) and integrated together to get the functionality of three bit flash ADC. The high speed three bit flash ADC is designed, simulated and verified in CADENCE Design Environment using $180 \mathrm{~nm}$ CMOS technology with a power supply of $1.8 \mathrm{~V}$.

\section{IV.DESIGN OF THE COMPARATOR USING OTA}

A straightforward approach to make a comparator is to design a high gain amplifier with differential analog input and single-ended large swing output. This kind of comparator is also called open-loop comparators. This comparator consists of the differential input single output OTA connected to an inverter as shown in figure 3. 


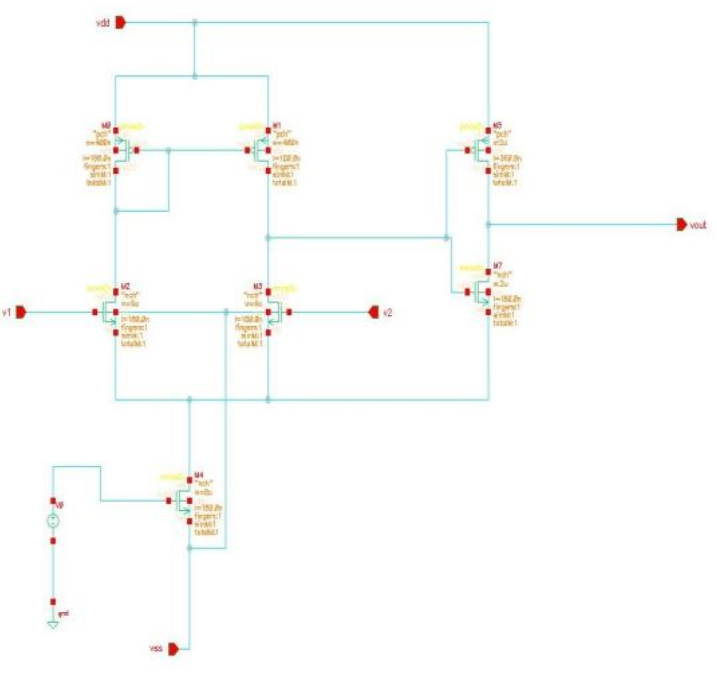

Fig. 3A Comparator circuit Schematic

A. Transient Analysis: A sine wave of $10 \mathrm{MHz}$ frequency, $500 \mathrm{mV}$ p-p is given as input to comparator is shown in fig. 4 . When the input is greater than reference the output is HIGH and the output is LOW when input is lesser than the reference.

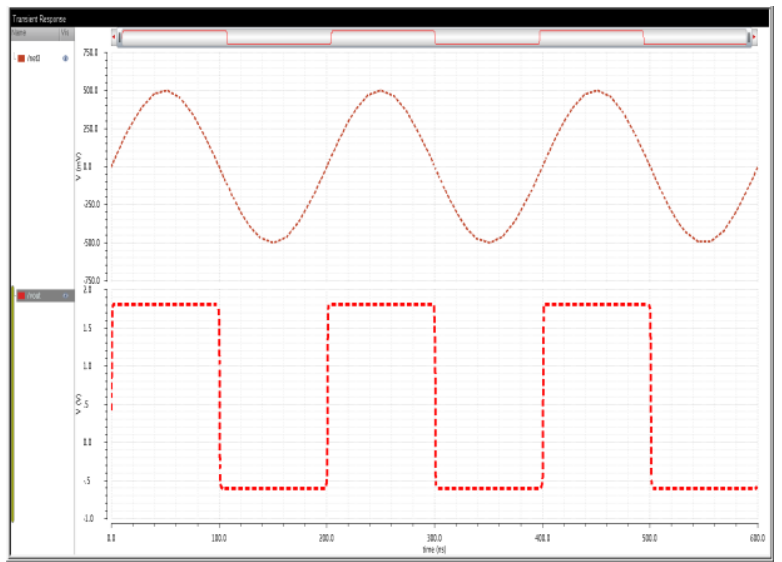

Fig.4 Transient Analysis of a Comparator

The AC response is as shown in the fig. 5. The DC gain of the comparator is $56 \mathrm{~dB}$ and Unity Gain Bandwidth is $2.5 \mathrm{GHz}$.

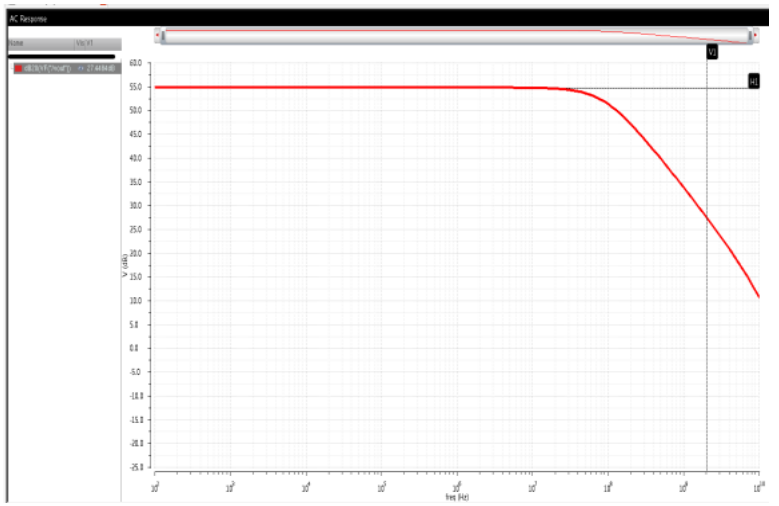

Fig. 5 AC Response of the Comparator
TABLE I PERFORMANCE SUMMARY OF COMPARATOR

\begin{tabular}{|c|c|}
\hline Parameter & Proposed Comparator \\
\hline Technology Size & $180 \mathrm{~nm}$ \\
\hline Operating Freq. & $10 \mathrm{MHz}$ \\
\hline DC Gain & $56 \mathrm{~dB}$ \\
\hline UGB & $2.5 \mathrm{GHz}$ \\
\hline
\end{tabular}

\section{ENCODER DESIGN FOR FLASH ADC}

Flash ADC comprises of three parts; resistor ladder, comparator and thermometer code to binary code converter. $\mathrm{N}$ bit flash ADC architecture requires $2^{\mathrm{N}}-1$ comparators for its operation. The reference voltage is generated with the help of $2^{\mathrm{N}}$ equally sized resistor which constitutes resistor ladder [14]. Since the comparators are working in parallel, flash ADC completes its conversion in one cycle. The output of the comparators is coming in a specific manner which is called thermometer code. The thermometer code is converted into binary code with the help of thermometer code to binary code conversion. The speed of the converter plays a crucial role in the design of flashADC.

Thermometer to Binary encoder is taken as a choice of design which compresses the comparator output having multiple binary inputs into small number of digital output [12]. This encoder depends on the thermometer code which is produced by the array of comparators. It outputs the binary value representing highest position of active input. The logic showing the Thermometer to Binary encoder design for 3 bit output is shown in table 2 . The output equation which is obtained using the logic is given below. Using K'map technique below equations are derived-

$$
\begin{gathered}
\mathrm{B} 0=\mathrm{T} 3 ; \\
\mathrm{B} 1=\mathrm{T} 1+\mathrm{T} 3 \mathrm{~T} 4+\mathrm{T} 4 \mathrm{~T} 5 ; \\
\mathrm{B} 3=\mathrm{TOT} 1+\mathrm{T} 1 \mathrm{~T} 2+\mathrm{T} 3 \mathrm{~T} 4+\mathrm{T} 5 \mathrm{~T} 6 .
\end{gathered}
$$

\section{TABLE II THERMOMETER TO BINARY ENCODER LOGIC TABLE}

\begin{tabular}{|l|l|l|l|l|l|l|l|l|l|}
\hline T0 & T1 & T2 & T3 & T4 & T5 & T6 & B0 & B1 & B2 \\
\hline 0 & 0 & 0 & 0 & 0 & 0 & 0 & 0 & 0 & 0 \\
\hline 0 & 0 & 0 & 0 & 0 & 0 & 1 & 0 & 0 & 1 \\
\hline 0 & 0 & 0 & 0 & 0 & 1 & 1 & 0 & 1 & 0 \\
\hline 0 & 0 & 0 & 0 & 1 & 1 & 1 & 0 & 1 & 1 \\
\hline 0 & 0 & 0 & 1 & 1 & 1 & 1 & 1 & 0 & 0 \\
\hline 0 & 0 & 1 & 1 & 1 & 1 & 1 & 1 & 0 & 1 \\
\hline 0 & 1 & 1 & 1 & 1 & 1 & 1 & 1 & 1 & 0 \\
\hline 1 & 1 & 1 & 1 & 1 & 1 & 1 & 1 & 1 & 1 \\
\hline
\end{tabular}

The outputs of comparators form a thermometer code is a combination of a series of zeros and a series of ones, e.g., $000 \ldots 011 \ldots 111$. Because binary code is usually needed for digital signal processing, a thermometer code is then transformed to a binary code through a (2k-1)-to-k 
Thermometer to Binary encoder, where $\mathrm{k}$ is the resolution (bits) of ADCs.

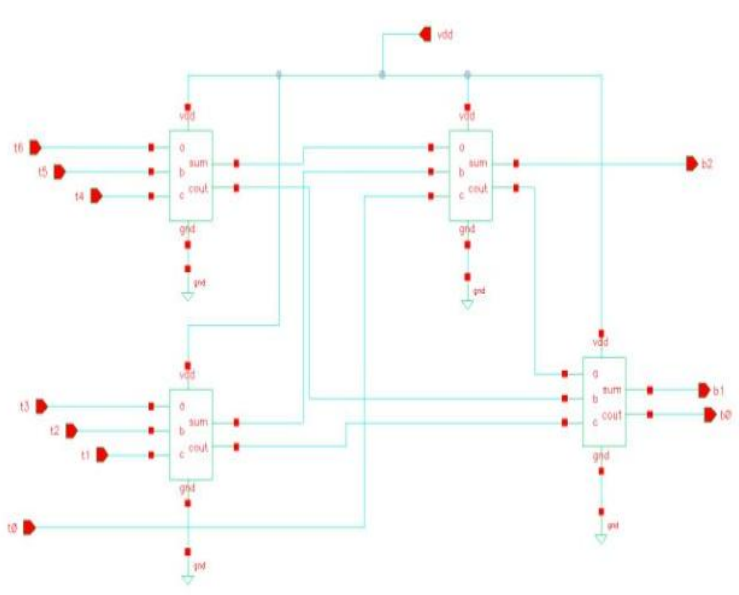

Fig. 6 Thermometer to Binary Encoder

The circuit of the Thermometer to Binary Encoder is shown in Fig. 6. The circuit is simplified to four full adders are connected as shown in the Fig above. The full adders are designed using half adders to simplify the circuit further.

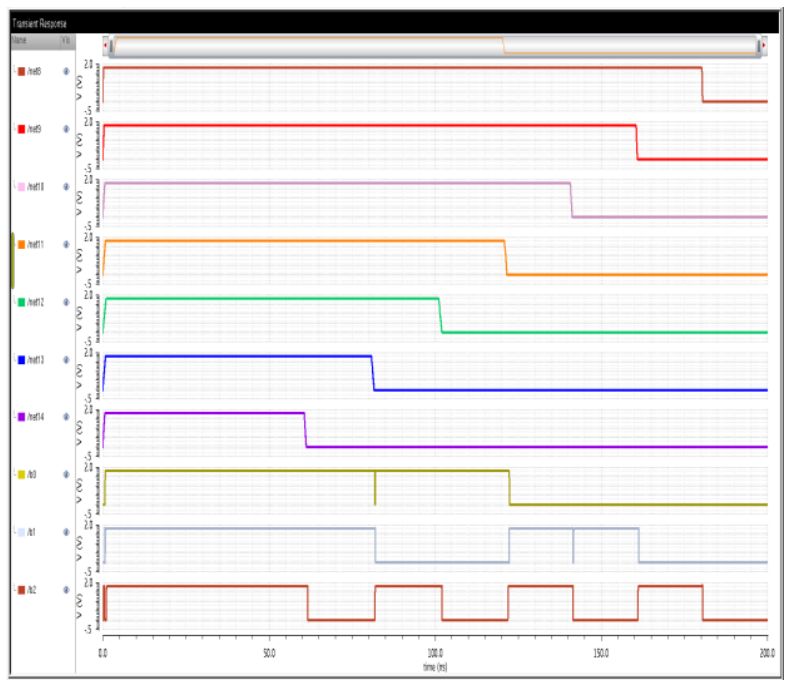

Fig.7 Transient Response of Thermometer to Binary Encoder

The output of the Thermometer to Binary Encoder is shown in Fig. 7 and for all the input combinations a desired output is obtained. When the input to the thermometer to binary encoder is 0000000 , the output is 000. Similarly, when the input to the encoder is 0000001 , the output is 001 and this continues until the input to the encoder is 1111111 , and the output is 111 .

The transient response of the compete flash ADC for a frequency of $1 \mathrm{MHz}, 3 \mathrm{MHz}, 5 \mathrm{MHz}$, and $10 \mathrm{MHz}$ are shown in figures (8-11). For the given analog input, all the eight combinations from 000 to 111 are observed.

\section{A. For 1 MHz:}

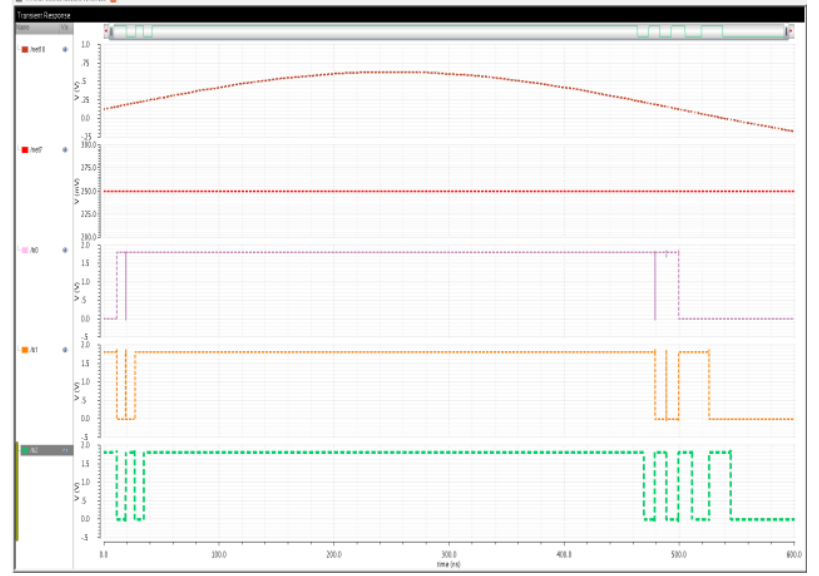

Fig.8 Three Bit Flash ADC transient response for a frequency of $1 \mathrm{MHz}$

\section{B. For 3 MHz:}

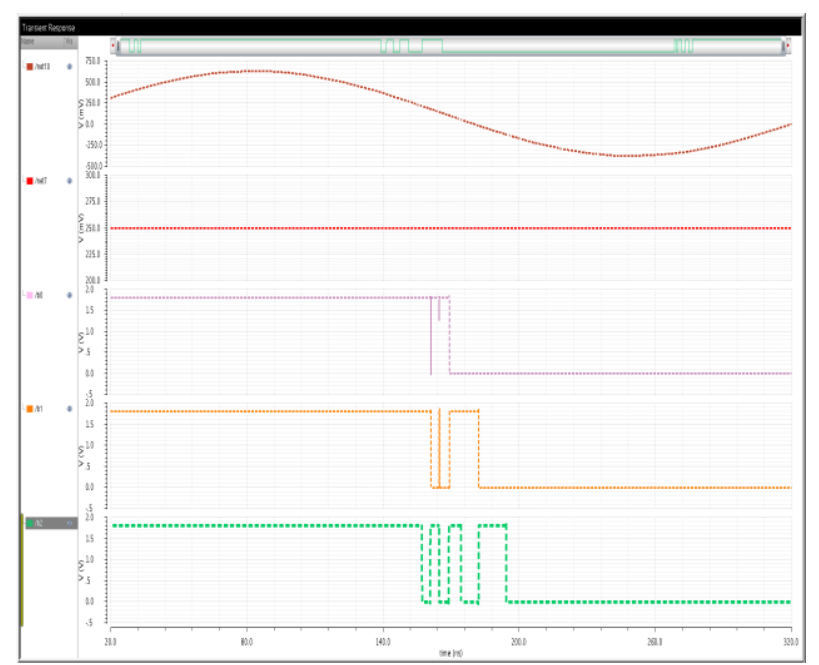

Fig. 9 Three Bit Flash ADC transient response for a frequency of $3 \mathrm{MHz}$

\section{For 5 MHz:}

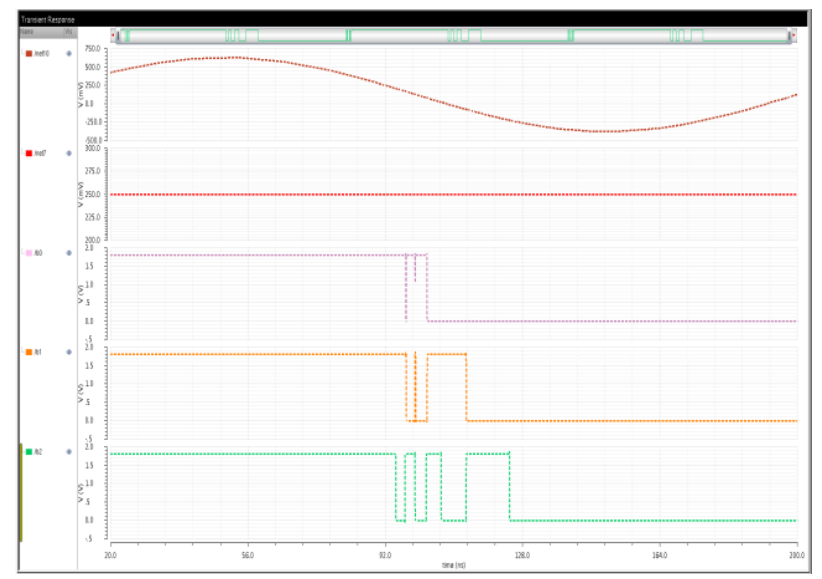

Fig.10Three Bit Flash ADC ADC transient response for a frequency of $5 \mathrm{MHz}$ 


\section{For 10 MHz:}

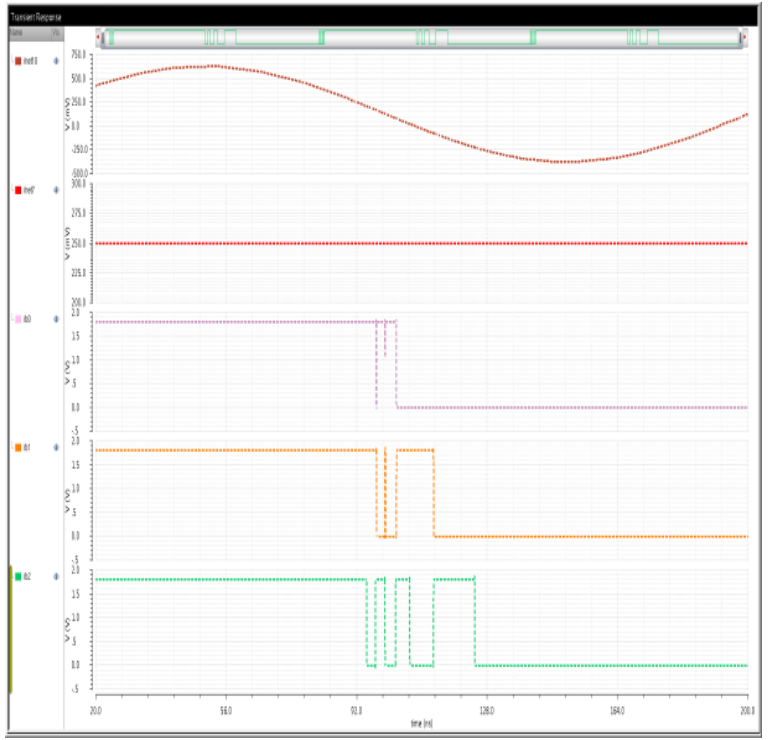

Fig. 11 Three Bit Flash ADC transient response for a frequency of $10 \mathrm{MHz}$

The transient response of the compete flash ADC for a frequency of $10 \mathrm{MHz}$ is shown in fig. 8 . For the given analog input, all the eight combinations from 000 to 111 are observed.

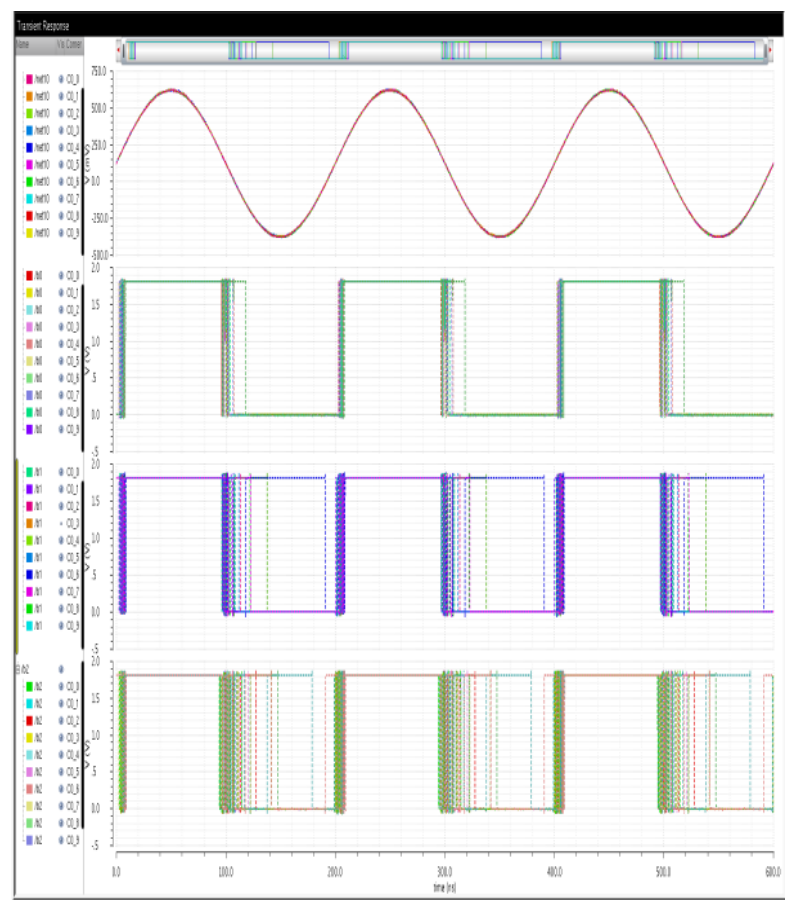

Fig.12 Corner analysis of three bit flash ADC

The corner analysis of three bit flash ADC is shown in fig. 9. For the given analog input, all the eight combinations from 000 to 111 are observed at all the corners. The response of all corners is same indicating the there is no variation in corners and hence the proposed designed meets all the specific requirements.

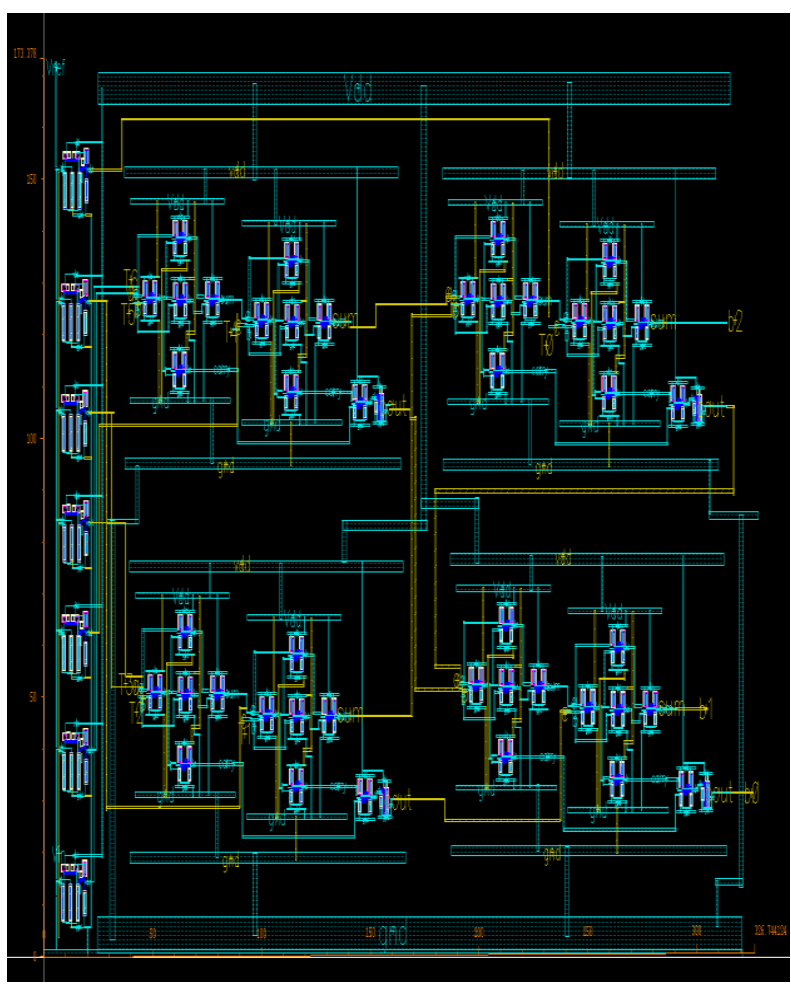

Fig. 13 Layout of Flash ADC

Area of Flash $\mathrm{ADC}=326.7 \mu \mathrm{m} \times 273.3 \mu \mathrm{m}$ $=0.089287 \mathrm{~mm}^{2}$.

\section{VI.CONCLUSION}

Modern communication systems need higher information rates that have increased the demand for the high speed transceivers. For a system to workwith efficiency, all blocks of that system ought to be quick. It are often seen that the analog interfaces are main bottleneck within the whole system in terms of speed and power. This fact has emphasize researchers to develop and implement high speed analog-to- digital converters (ADCs) with low power consumption.

This paper demonstrates a high speed three bit flash ADC used for Wireless LAN applications. The designed converter is a practical approach targeted at low power high speed converter for wireless applications. This design is a flash based ADC converter with a finite output resolution of three bits and power consumption about $223 \mathrm{uW}$ and occupies a chip area of $0.089287 \mathrm{~mm}^{2}$.The high speed flash ADC is being designed and verified using CADENCE Virtuoso tool with CMOS $180 \mathrm{~nm}$ technology.

\section{ACKNOWLEDGMENT}

We sincerely thank the management, the principal and ECE dept. staff of CMR Engineering College, Hyderabad for encouraging in this work. Lastly, we thank almighty, our parents, friends for their constant encouragement without which this paper would not be possible. 


\section{REFERENCES}

[1] Biswas, Suman, Jitendra Kumar Das, and Rajendra Prasad. "Design and implementation of 4 bit Flash ADC using low power low offset dynamic comparator", International Conference on Electrical Electronics Signals Communication and Optimization (EESCO), 2015.

[2] Hosur K. N., Dariyappa, Shivanand, Vijay, Nagesha, Girish V. Attimarad, and Harish M.Kittur. "Design of 4 bit flash ADC using TMCC \& NOR ROM encoder in 90nm CMOS technology", 2015 International Conference on Trends in Automation Communications and Computing Technology (I-TACT-15), 2015.

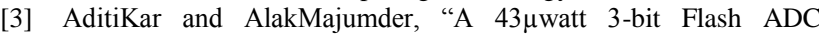
designed with TMCC and Bit Referenced Encoder in $180 \mathrm{~nm}$ CMOS Technology", International Journal of Advanced Science and Technology Vol.79 (2015), pp.49-60.

[4] Zhao, Xiao, Huajun Fang, and Jun Xu. "A LOW POWER CURRENT RECYCLING CONSTANT-gm RAIL-TO-RAIL OTA", Journal of Circuits Systems and Computers, 2014

[5] T.Maneesha, T.S GhouseBasha, "Design Of Low Voltage low Power Inverter based comparator using CMOS technology", International Journal of emerging Engineering Research and Technology(IJEERT), Volume 2, September 2014

[6] Lee. Y.Chong "Design of an optimized low power and high speed 3 bit flash Analog-to-Digital Converter (ADC) using $45 \mathrm{~nm}$ technology" Third International Conference on Advanced Computing and Communication Technologies (IACCT), 2013,pp.307-311.

[7] DhrubajyotiBasu, Sagar Mukherjee , "An optimized analog layout for a Low Power 3-bit flash type ADC modified with the CMOS inverter based comparator designs", International Conference on Circuits, Power and Computing Technologies (ICCPCT), 2013.

[8] Sujeet Mishra, Balchand Nagar, "Design of a TIQ comparator for high speed and low power 4-bit Flash ADC", International Journal of Emerging Technologies in Computational and Applied science (IJETCAS), 2013

[9] Marcel Siadjinenjinowa, "Design of low power 4-bit flash ADC based on standard cells", New Circuits and Systems Conference (NEWCAS), 2013, IEEE.

[10] M Subba Reddy, S. TipuRahaman, "An effective 6-bit flash ADC using low power CMOS technology", Advanced Computing Technologies (ICACT), 2013, IEEE

[11] Arunkumar P Chavan, An Efficient Design of 3bit and 4bit Flash ADC, International Journal of Computer Applications Volume 61No. 11, January 2013.

[12] SmritiSubhanad, Dr. H.P Shukla, A.G Rao, "Design and Simulation of a high speed CMOS Comparator", International journal Electronics and Communication Engineering, ISSN 0974-2166 Volume 6, 2013.

[13] Varghese, George Tom, and K. K. Mahapatra. "A high speed low power encoder for a 5 bit flash ADC", 2012 International Conference on Green Technologies (ICGT), 2012.

[14] P.Rajeswari, R.Ramesh, A.R.Ashwatha 2012," An approach to design Flash Analog to Digital Converter for High Speed and Low power Applications", International Journal of VLSI design \& Communication Systems (VLSICS) Vol.3, No.2.

[15] Ili Shairah Abdul Halim, NurulAisyah Nadia BintiZainalAbidin, Ezra AfhzanAb Rahim, "Low power CMOS charge sharing dynamic latch comparator using $0.18 \mu \mathrm{m}$ technology", IEEE Regional symposium on micro and nano electronics (RSM),pp 156160, Sep 2011.

[16] Pradeep Kumar, AmitKolhe, "Design \& Implementation of Low Power 3-bit Flash ADC in 0.18um CMOS", International Journal of Soft Computing and Engineering (IJSCE), ISSN: 2231-2307, Volume-1, Issue-5, November, 2011.

[17] A. Mahesh Kumar, VenkatTumamala, "Design of a Low Power, VariableResolution Flash ADC", 22nd International Conference on VLSI Design, 2009, IEEE.

[18] M. Vesterbacka. "6 bit $1 \mathrm{GHz}$ CMOS silicon-oninsulator flash analog-to-digital converter for read channel applications", Proceedings of the European Conference on Circuit Theory and Design 2005.
[19] Sung-mo Kang, Yusuf Leblebici, "CMOS Digital Integrated Circuits: Analysis and Design", third edition, Tata McGraw-Hill edition 2003.

[20] R.Baker, H.W.Li, and D.E. Boyce, CMOS Circuit Design, Layout and Simulation. Prentice Hall 2000.

\section{BIOGRAPHIES}

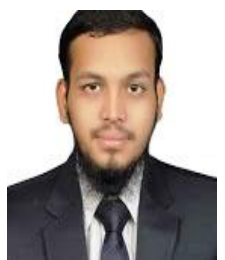

MirzaNemath Ali Baig is currently working as Assistant Professor in ECE dept. of CMR Engineering College, Hyderabad, India. He has completed hisM.E. fromMuffakhamJah College of Engineering and Technology, Hyderabad with the specialization of Digital Systems. He has kin interest to do research in VLSI and signal processing.

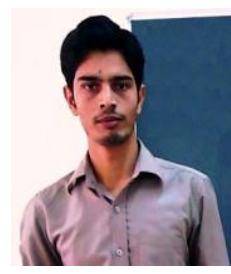

Rakesh Ranjan is currently working as Assistant Professor inECE dept. of CMR Engineering College, Hyderabad, India. He has completed his M.Tech from National Institute of Electronics \& Information Technology, Aurangabad, Maharashtra with the specialization of Electronics Design and Technology. He is doing research in signal processing, Embedded System and VLSI 